\title{
KaVa Tutor: autorregulación y estimulación de procesos académicos en plataformas educativas.
}

\author{
Diego Samir Melo-Solarte ${ }^{1}$, Alvaro Joffre Uribe ${ }^{2}$ \\ ${ }^{1}$ Facultad de Ciencias e Ingeniería \& Centro de Educación a Distancia \\ Universidad de Manizales \\ Manizales - Caldas - Colombia \\ ${ }^{2}$ Facultad de Ingeniería \\ Universidad Militar Nueva Granada \\ Manizales - Caldas - Colombia \\ mdiego@umanizales.edu.co, alvaro.uribe@unimilitar.edu.co
}

\begin{abstract}
This paper sets out the design of web interfaces that consider affective and emotive characteristics to support teaching and learning processes in a distance academic program. Its bases are self-regulation and stimulation from the teachers in the preparation of the didactic material to be used in the course, as those from the students in its development. This project uses as a referent the Organizational Semiotics, which allows the consideration of social, pragmatic, and semantic aspects, besides the infrastructure. In this way, the boarding of the problem is done with wider meanings.
\end{abstract}

Resumen. Este artículo plantea el diseño de interfaces web que contemplen características afectivas y emotivas para apoyar los procesos de enseñanza y aprendizaje de un programa académico a distancia, tomando como base la autorregulación y la estimulación de los docentes en la preparación del material didáctico a ser usando en un curso, como de los estudiantes en el desarrollo del mismo. Este proyecto usa como referente base a la Semiótica Organizacional, lo cual permite considerar aspectos sociales, pragmáticos, semánticos, además de su infraestructura, lo cual se traduce en un abordaje del problema con una mayor amplitud de significados.

\section{Introducción}

Este proyecto de investigación está orientado a evaluar las interfaces en los sistemas usados como mediadores de los procesos académicos para los cursos que se ofertan en la modalidad de Educación a Distancia $(\mathrm{EaD})$ por Instituciones de Educación Superior IES. Bajo este proceso evaluativo se busca pesquisar alternativas gráficas, comunicativas e interactivas que contribuyan en el desarrollo de los procesos académicos y estimulen la ejecución de los mismos.

Básicamente, este proyecto involucra a dos actores principales que son: docentes, con la construcción del material académico a ser usado en un curso y estudiantes, con el desarrollo de los cursos usando la mediación denominada "módulos académicos"; de esta manera, con los docentes se pretende construir interfaces de usuario que guíen el

Investigación realizada con el apoyo de la unión temporal "Innovación Tecnológica UManizales - Chec" y el Departamento Administrativo de Ciencia, Tecnología e Innovación (Colciencias) 
proceso de construcción de los módulos o cursos académicos de calidad, estimule la producción escrita del docente y genere un marco de autorregulación en cuanto a tiempo de producción y calidad del producto; de igual forma, para los estudiantes se pretende construir interfaces que guíen el proceso de aprendizaje, estimulen y controlen el desarrollo de actividades de aprendizaje, y adicionalmente permitan generar mecanismos que promuevan la autorregulación en cuanto a la responsabilidad y cumplimiento de las actividades planteadas en el desarrollo de un programa a distancia.

Es claro que tanto los procesos de producción docente como los procesos de aprendizaje de un estudiante, requieren un seguimiento y control permanente, sin embargo, cada vez es más difícil que los encargados de coordinar este proceso, puedan asumir esta dispendiosa actividad en su plenitud, por esta razón se busca que las herramientas tecnológicas usadas como mediadores del proceso realizado por cada actor, también puedan ser usadas como mecanismos de autorregulación, autocontrol y concientización, teniendo una visión panorámica del proceso, la evolución del mismo y su comparación frente a otros actores.

Las áreas que fuertemente soportan el desarrollo de este proyecto, se centran en la disciplina de la Interacción Humano Computador (IHC) con el uso de la Semiótica Organizacional (SO), la cual se determina como la comprensión y relación que se da entre el ser humano y un artefacto digital, ocupándose del diseño, evaluación e implementación de sistemas informáticos interactivos, considerando las cualidades de los usuarios. Por otra parte también se contempla el uso de la Ingeniería de Software con el uso de algunas metodologías ágiles para el desarrollo de aplicativos, que permitan guiar la producción de una manera rápida y efectiva.

En muchas ocasiones se habla que el análisis de un sistema de información debe ser evaluado integralmente, sin embargo muchos modelos inclinan su estudio desde una perspectiva puramente técnica y operativa, dejando de lado al usuario como un agente activo dentro del proceso, y donde este agente está expuesto a una serie de influencias que puede afectar la interacción y la vez los resultados del sistema; es de esta manera que la SO busca crear un nuevo panorama del análisis de los sistemas de información basados en la comunicación como eje central de la interacción y donde las interpretaciones de los signos toman relevancia.

La SO se interpreta como una disciplina que estudia los procesos organizacionales, teniendo como base la naturaleza, características, funciones y efectos de la información y la comunicación en un contexto organizacional [Stamper et al., 1993; Liu, 2000] tomado de [Neris,2010]. Desde la perspectiva de la SO, una organización puede ver a la información como un sistema donde los agentes emplean los signos para realizar unas acciones o propósitos específicos [Neris, 2010].

Son varias herramientas metodológicas que hacen parte de la SO, si consideramos la Cebolla Semiótica [Liu, 2000] es posible analizar cualquier sistema de información a nivel técnico, formal e informal, lo cual permite tener diferentes perspectivas de estudio, logrando una visión integral del sistema sin dejar de lado aspectos sociales.

Por otra parte, se encuentra un complemento propuesto por Stamper a través conjunto de métodos para apoyar el análisis de un sistema de información desde la perspectiva de la SO llamados MEASUR (Methods for Eliciting, Analysing and Specifying Users Requirements) [Liu, 2000]. Entre los cuales tenemos el Método de Articulación de Problemas (PAM) que permitirá evaluar un sistema de información como un problema 
desde sus estados iniciales de entendimiento, cuando sus fronteras aún no son claras y la solución debe considerar los aspectos sociales, pragmáticos y semánticos involucrados, además de la infraestructura necesaria (aspectos físicos, empíricos y sintácticos) [MeloSolarte \& Baranauskas, 2009].

Por otra parte tenemos los métodos ágiles como una respuesta a la gran demanda por métodos y modelos de procesos que sean flexibles a los constantes cambios en el desarrollo de un determinado proyecto de software, y a la entrega rápida del mismo, sin dejar de lado la calidad.

En la actualidad se encuentra una gran cantidad de métodos ágiles disponibles a la orden del día, estos métodos pueden variar de manera significativa, por tal razón es necesario escoger cuidadosamente de acuerdo a las características del proyecto de software a ser desarrollado, algunos de los principales ejemplos de métodos ágiles y más populares tenemos [Koch, 2005]: Adaptive Software Development (ASD), Dynamic System Development Method (DSDM), eXtreme Programming (XP), Scrum y AIPM [Hornung et al, 2013]: un modelo de proceso ágil desarrollado en el contexto del proyecto [ECidadania, 2013], este modelo busca integrar las metodologías ágiles de desarrollo de software conjuntamente con técnicas de tomadas de la Interacción Humano Computador, la semiótica Organizacional, el Diseño Universal, el trabajo colaborativo, entre otros.

Si bien es cierto que existen muchas metodologías ágiles para el desarrollo de software, es importante reconocer que estas metodologías no se convierten en "camisas de fuerza" que requieran ser aplicadas con rigor, antes por el contrario, el tratado de estas metodologías buscar orientar el desarrollo de software brindando libertad de actuación según las necesidades de los proyectos o de los mismos usuarios.

\section{Metodología}

Para el desarrollo de este proyecto, se ha conformado una metodología basada en las técnicas de los modelos ágiles de desarrollo de software y adicionalmente se integra con las técnicas de trabajo de la Interacción Humano Computador, el objetivo es crear un esquema de trabajo ligero y focalizado en el esquema de operación del usuario final.

Las metodologías ágiles permiten abordar el desarrollo de software de una manera práctica y más rápida, creando una perspectiva paralela a los ciclos tradicionales de la Ingeniería de Software, sin embargo se conserva el rigor y la calidad del producto final y el cumplimiento de los requerimientos. Por otra parte, la Interacción Humano Computador pone como eje central de un desarrollo de software al usuario final, involucrando sus capacidades o restricciones físicas o cognitivas; además de otros elementos que de una $\mathrm{u}$ otra forma inciden en su interacción con un sistema computacional, como son sus conocimientos previos, sus habilidades, su cultura, su entorno social, entre otras.

Para el desarrollo de este proyecto el proceso de investigación es transversal a lo largo del todo el proyecto, y se enfoca en el estudio y aplicación de mecanismos de comunicación basados en Awareness orientada a sistemas colaborativos [Almeida \& Baranauskas, 2008], esto busca diseñar los elementos de comunicación orientados estimular y autorregular la producción de los docentes y el trabajo de los estudiantes. Adicionalmente, el diseño y construcción de los aplicativos, se ejecutan en tres fases: 


\section{Fase 1: Análisis del proceso de construcción de módulos académicos y la ejecución de un curso.}

En esta fase se evalúa los requerimientos, las características y la viabilidad de un sistema que pueda dar soporte al proceso de construcción de módulos académicos para apoyar las actividades de los programas ofertados en una modalidad a distancia, adicionalmente, se evalúa la manera como se desarrolla un curso con la intervención de docentes, tutores y estudiantes.

Los requerimientos generales son levantados bajo el esquema de entrevista y observación, sin embargo el análisis y la especificación de requerimientos se acompaña de la Semiótica Organizacional con el uso del PAM y las herramientas: Partes Interesadas, Cuadro de Evaluación y Framework Semiótico; como mecanismo para evaluar y articular problemas, integrando a todo el grupo de producción (experto temático, unidad pedagógica, unidad gestión tecnológica, unidad de comunicación y medios, unidad administrativa, equipo docente y estudiantes), enfocando el sistema a las necesidades reales y características operativas de los usuarios finales.

Esta técnica de trabajo pretende lograr una buena caracterización del problema desde perspectivas no solo técnicas, sino también sociales, económicas y culturales; mientras que paulatinamente se van visibilizando soluciones que pueden tornarse factibles a la hora de crear un diseño. Una vez caracterizado el problema se procede a realizar el análisis estructurales y funcionales del sistema de información, tratando de identificar una arquitectura dinámica y flexible que permita el crecimiento progresivo del sistema.

El análisis involucra la revisión literaria sobre mecanismos de comunicación a través de "Awareness" para percibir o reconocer esquemas de trabajo colaborativos y patrones sensoriales que permitan estimular, provocar, conocer el entorno y el estado de la construcción de módulos académicos.

\section{Fase 2: Diseño de la interacción y prototipado no funcional}

El diseño del sistema se enfoca en la interacción que debe tener el usuario final, la amigabilidad de las interfaces, la versatilidad con la que se puede realizar los procesos y la contribución para orientar a los diferentes actores.

El diseño se enfoca en dos elementos primordiales, la interacción a través de interfaces web y la arquitectura de modular del sistema de información que permita garantizar la evolución de funcionalidades del sistema. De esta manera, previo al diseño de interfaces no funcionales (mockups), se aprovecha el análisis hecho sobre los actores en el cual se ha identificando sus características operativas, sus restricciones y sus habilidades, para poder maquetar una estructura de interacción y comunicación práctica de ser implementada, instalada y administrada; pero ante todo que brinde al usuario final una accesibilidad y usabilidad de los recursos lo más próximo de su intuición.

El diseño de las interfaces web y la construcción de un prototipo no funcional se desarrolla bajo la técnica braindrawing, y tiene por objetivo implementar esquemas de comunicación indirecta como "agentes estimuladores" o afectivos, caracterizando un fenómeno afectivo como un estado reconocido por un conjunto de disposiciones psicológicas y biológicas ligada a la afectividad [Longaray, 2012], siendo que a nivel de interfaces deberán reflejar una espacio de trabajo que considera al usuario con sus cualidades, sus habilidades y sus emociones. 


\section{Fase 3: Valoración del diseño de las interfaces y prototipado no funcional}

El esquema de pruebas busca comprobar que los requerimientos planteados inicialmente estén acordes a las funcionalidades simuladas en las interfaces, y que adicionalmente se evalué las características de usabilidad y accesibilidad que debe tener cada interfaz. Para ello el plan de pruebas contemplará dos momentos, uno de confrontación de los requerimientos versus funcionalidades diseñadas y otro momento, la evaluación basada en el chequeo heurístico realizado por los usuarios. Estas pruebas se harán sobre cada una de las interfaces diseñadas.

Sobre el prototipo no funcional que contiene embebido las interfaces diseñadas, se plantea una prueba de integridad que simule el proceso completo de la construcción de un módulo, en este proceso serán involucrados actores externos e internos con el fin de determinar un concepto claro sobre lo complejidad y usabilidad del sistema.

El proceso metodológico tiene inmerso un esquema de trabajo inclusivo, el cual busca garantizar un abordaje que garantice que los productos y servicios proyectados estén atendiendo las necesidades del público de la forma más amplia, independiente de los conocimientos y las habilidades que los usuarios finales tengan. Por tal razón este proyecto busca conservar las características del diseño inclusivo en cuanto a: uso equitativo, teniendo en cuenta la diversidad de habilidades que pueden tener los usuarios; flexibilidad en el uso, considerando la capacidad para evitar que el usuario cometa errores; diseño simple, buscando un esquema de interacción intuitivo; información perceptible, es decir mantener al usuario constantemente informado sobre el estado del sistema y de su interacción; y menor esfuerzo para lograr interactuar con el sistema y lograr los objetivos deseados.

Finalmente, el esquema de trabajo inclusivo pretende estar constantemente valorando el diseño de interfaces frente a los posibles usuarios, escuchar al usuario final es una excelente forma de captar nuevas ideas que perfectamente pueden ser implantadas en el diseño de interfaces, alejando el concepto técnico y acercando el concepto empírico del usuario.

\section{Diseño y desarrollo de KaVa Tutor}

Una vez identificados los requerimientos del proyecto, se evidenció claramente dos entornos de trabajo a los cuales se les aplicará el mismo marco metodológico, son ellos: un entorno orientado a la producción docente, la cual está focalizada en construcción teórica del módulo por parte de un experto temático y el segundo entorno está orientado a la participación de los estudiantes y tutores a lo largo del desarrollo de un determinado curso. A continuación se describe los avances y alcances logrados con Kava Tutor.

\subsection{PAM en el análisis de requerimientos}

Con el uso de la Cebolla Semiótica y la participación de diferentes actores del proceso se logró caracterizar los dos entornos de trabajo; posteriormente con el uso de los artefactos del PAM se definió estrategias para poder analizar a fondo las características de un problema, considerando los elementos técnicos y sociales que implica la interpretación, análisis y resolución de un problema.

A través de estas técnicas ha sido posible identificar las diferentes "partes interesadas", es decir, los elementos que de forma directa o indirecta se ven afectados o influenciados por el problema como un todo; el reconocer las partes interesadas ayuda a interpretar el 
problema no como una cuestión meramente operativa sino como un sistema que se ve afectado por un entorno; en la Figura 1 se puede observar la primera versión de las partes Interesadas del proyecto.

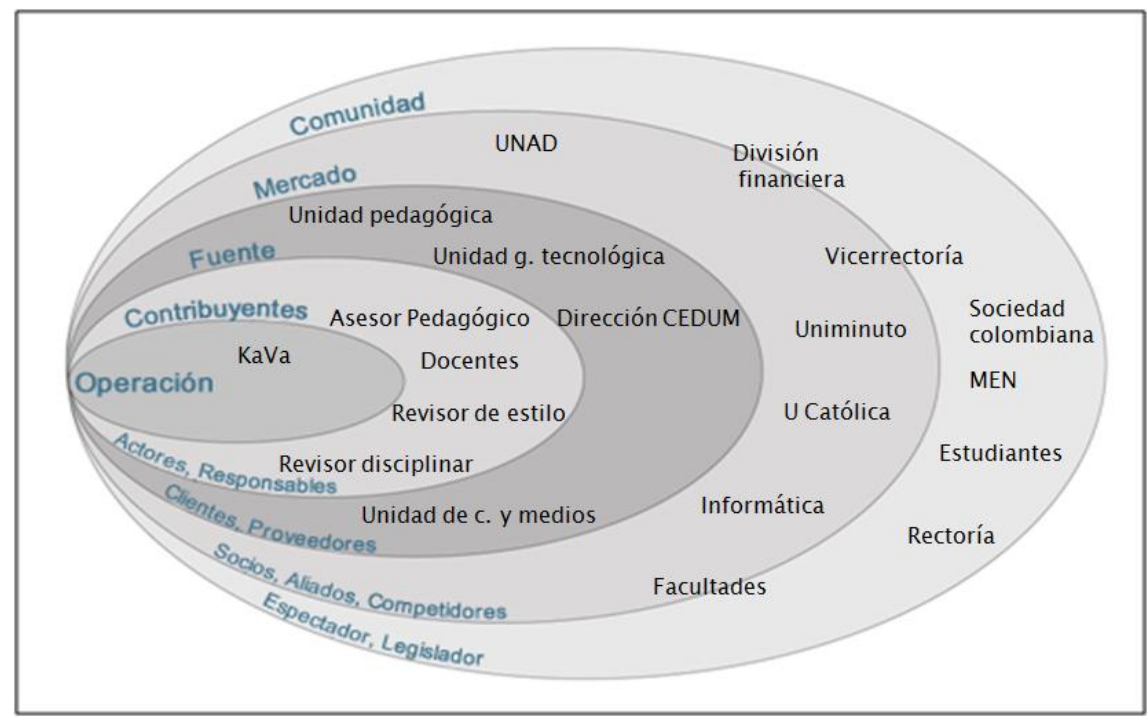

Figura 1: Partes Interesadas proyecto KaVa

Identificar los actores o responsables permite refinar la construcción el modelo de funcional, identificar los clientes o proveedores determina los integrantes que se encargarán de proporcionar el servicio, a su vez que conocer los socios, aliados o los competidores ayuda a realizar un beanchmarking que permita evaluar a la competencia y la manera como ellos realizan los mismos procesos, a fin de incorporar soluciones que puedan ser adaptables; finalmente, es necesario resaltar que los sistemas de información de una u otra forma se ven afectados por la sociedad, es por esto que siempre tiene que estar sujeto a la reglamentación institucional y a su vez a la nacional.

Las Partes Interesadas identificadas en el proyecto fueron analizadas y estudiadas con el apoyo de otros artefactos como el Cuadro de Evaluación o el Framework Semiótico, tratando de profundizar en algunos temas como la intervención de actores externos como revisores (disciplinar o estilo), los tiempos de producción, la calidad de la producción, las orientaciones para abordar la construcción de un módulo, los procesos de los tutores en la ejecución de un curso, la dedicación de un estudiante al proceso de aprendizaje, elementos que motivan al estudiante en sus actividades, entre otros.

\subsection{Modelo de producción de módulos}

Un primer paso para el diseño del sistema KaVa Tutor ha sido el análisis y adecuación del modelo de construcción y producción de módulos académicos identificando dos momentos, el primero orientado a la construcción de contenidos temáticos y el segundo momento orientado a la producción del material multimedia para ser montado en la Plataforma Educativa que usa la institución (Moodle 2.3).

La Figura 2 muestra la división del primer momento de la construcción dividido en 3 fases, para que permitan la producción de los contenidos de forma gradual y generar periodos de corte que a su vez permitan determinar la evolución del módulo. 


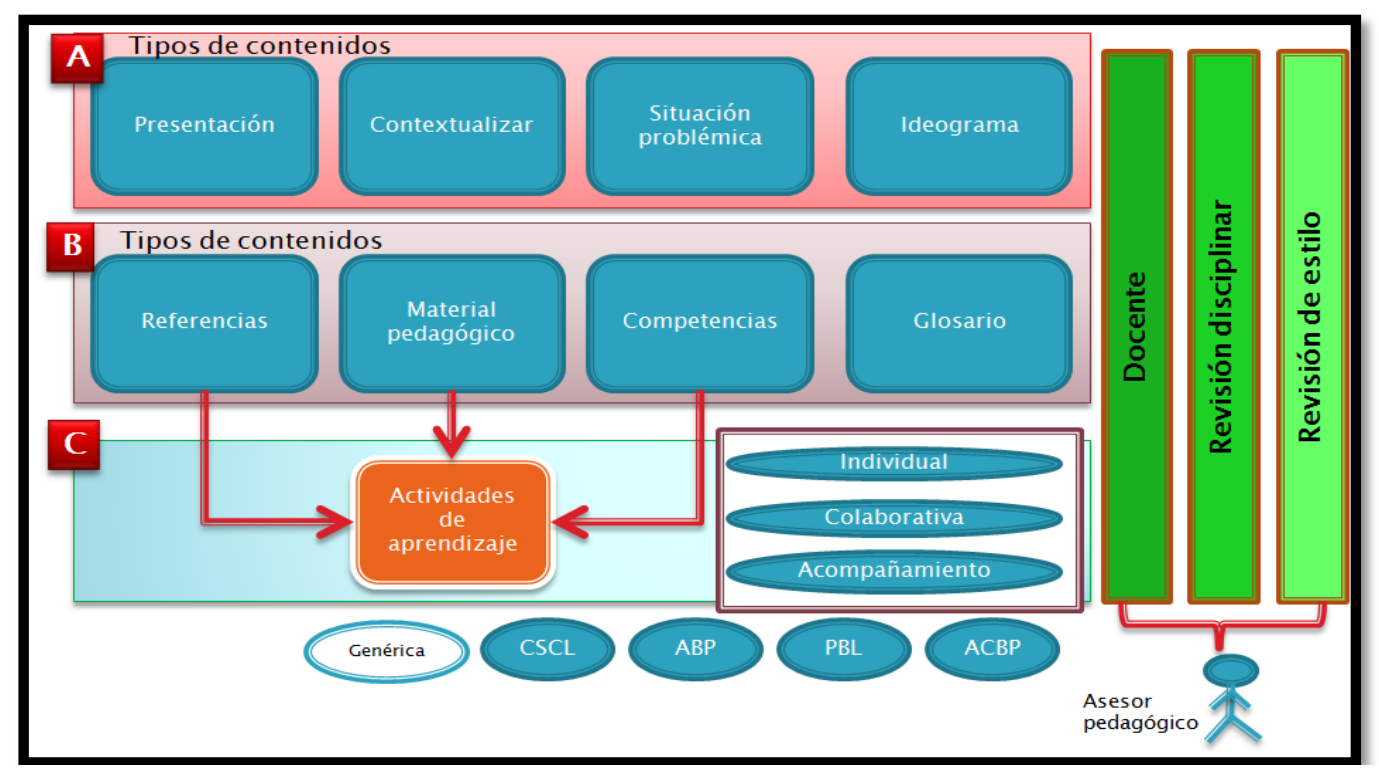

Figura 2: Elaboración de módulos académicos - Modelo: Fase de construcción

Fase A: compuesta por los contenidos presentación, contextualización, situación problémica e ideograma, teniendo en cuenta que estos contenidos no tienen dependencia de otros contenidos, además están orientados a contextualizar el módulo.

Fase B: esta fase está compuesta por cuatro contenidos referencias, material pedagógico, competencias y glosario, siendo una fase orientada a determinar los objetivos del módulo y el material a ser usado.

Fase C: esta fase contiene únicamente el contenido denominado actividades, es decir se enfoca en la construcción de acciones que tendrá que desarrollar el estudiante para el logro de los objetivos propuestos.

En el primer momento intervienen los docentes, el asesor pedagógico, el revisor disciplinar y el revisor de estilo, siendo el asesor pedagógico quien pueda determinar cuándo un contenido puede pasar a la intervención de los otros actores. Cabe aclarar que en este proceso surgen algunas actividades que no están descritas en el modelo pero hacen parte de la dinámica que se requiere para llevar a un feliz término la producción de contenidos y su respectiva publicación.

El segundo momento en el proceso de construcción del módulo está orientado a un proceso creativo donde se busca que la parte textual del módulo esté acompañada por recursos tecnológicos y didácticos, de allí que la integración de las diferentes unidades del equipo de producción y el diálogo con el docentes sea fundamental para generar ideas que puedan materializarse y convertirse en ayudas pedagógicas para los estudiantes; el segundo momento también está compuesto por tres fases que son:

Fase D: se considera un momento creativo donde se determinan los elementos didácticos (multimedia) que acompañarán el módulo y además donde se genera un cronograma sobre el plan de montaje.

Fase E: esta fase está orientada a colocar todos los recursos y contenidos en la Plataforma Educativa bajo un estilo visual e interactivo previamente propuesto. 
Fase F: la fase final de la construcción está orientada a la revisión final del módulo por parte del docente y del coordinador del programa.

El foco del proyecto se centra en las funcionalidades del sistema desde el punto de vista de la interacción y el diseño de las interfaces de usuario, por tal razón fue diseñado un esquema de interacción para la producción de un módulo donde el docente inicia la construcción de los diferentes tipos de contenidos apoyados en la orientación del asesor pedagógico, los contenidos son revisados desde el punto de vista pedagógico. Una vez el asesor pedagógico estima que el módulo o algunos de sus componentes están correctamente construidos, son enviados a evaluación del revisor disciplinar. Finalmente, cuando el módulo es aprobado por el revisor disciplinar, interviene el asesor pedagógico para enviar el módulo al revisor de estilo, quién se encargará de hacer los ajustes de redacción necesarios.

\subsection{Implementación de interfaces}

Con la participación de diferentes actores se logró la creación de unas interfaces base, las cuales sirvieron de referente para ser perfeccionadas en sistemas computacionales al estilo mockups. El paso de mockups aprobadas a interfaces web se logró con la intervención de un diseñador visual dentro del equipo, el cual ha logrado materializar en las ideas en los diseños de interfaces a la vez que se cuida de los elementos gráficos e interactivos, la Figura 3 muestra el diseño de una interfaz que evolucionó del mockup a la implementación en web, pasando por las consideraciones de los usuarios las cuales fueron detectadas a través del uso de instrumentos de la SO.

Después de analizado los procesos de construcción de módulos académicos y el desarrollo de un curso, fue posible abstraer los elementos de autorregulación y estimulación que podían ser incorporados en las interfaces, manteniendo su usabilidad pero tornándolas más afectivas.

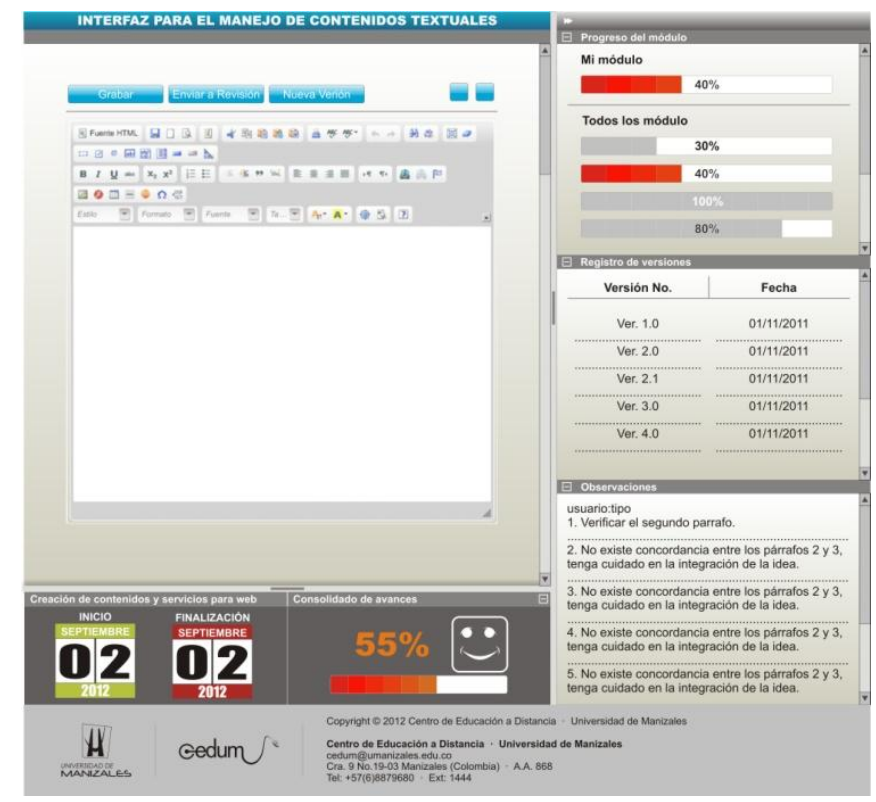

Figura 3: Interfaz para manejo de contenidos textuales

En la Tabla 1 se enuncian los principales elementos que han incorporado en las diferentes interfaces, cabe resaltar que se ha optado por el uso de emoticons y/o avatars a fin de reflejar el estado emocional del sistema como producto de la comparación del 
trabajo entre los diferentes actores, esto busca generar un efecto motivacional positivo sin trasgredir lineamientos pedagógicos.

\section{Tabla 1: Elementos visuales e interactivos incorporados en las interfaces de producción docente}

\begin{tabular}{|l|l|}
\hline \multicolumn{1}{|c|}{ Componente } & \multicolumn{1}{c|}{ Descripción } \\
\hline $\begin{array}{l}\text { Interfaz de seguimiento de } \\
\text { producción }\end{array}$ & $\begin{array}{l}\text { El docente y el estudiante pueden reconocer con colores y formas la } \\
\text { evolución de su producción }\end{array}$ \\
$\begin{array}{l}\text { Cuantificación de la } \\
\text { producción }\end{array}$ & $\begin{array}{l}\text { Un docente puede identificar porcentualmente su producción y visualizar } \\
\text { una comparación frente a otros docentes que están en el mismo proceso } \\
\text { para determinar su avance y su productividad }\end{array}$ \\
\hline $\begin{array}{l}\text { Producción frente a los } \\
\text { tiempos pactados }\end{array}$ & $\begin{array}{l}\text { Es posible identificar gráficamente el estado de la construcción de un } \\
\text { módulo frente a los tiempos acordados y/o promedio. }\end{array}$ \\
\hline Elementos de alerta & $\begin{array}{l}\text { Se han incorporado elementos visuales de alerta que permitan recordar al } \\
\text { docente posibles retrasos o comparar su producción con otros docentes } \\
\text { que están en el proceso. }\end{array}$ \\
\hline Registro de participación & $\begin{array}{l}\text { Se ha diseñado un algoritmo para identificar y cuantificar la intervención } \\
\text { de todos los actores en la producción de un módulo. }\end{array}$ \\
\hline $\begin{array}{l}\text { Incorporación de estándar } \\
\text { de colores }\end{array}$ & $\begin{array}{l}\text { Se adaptaron todos los iconos para reflejar con colores (gris, naranja, } \\
\text { verde, rojo) el estado de un proceso respectivamente (sin iniciar, } \\
\text { iniciado, terminado, retrasado) }\end{array}$ \\
\hline $\begin{array}{l}\text { Alerta de propiedad } \\
\text { intelectual }\end{array}$ & $\begin{array}{l}\text { Ha sido diseñado un algoritmo que permite comparar los textos creados } \\
\text { por un docente frente a textos disponibles en internet a fin de alertar } \\
\text { sobre posibles problemas de derechos de autor. }\end{array}$ \\
\hline Avance del curso & $\begin{array}{l}\text { Permite identificar la globalidad del curso y reconocer su avance frente a } \\
\text { los tiempos y actividades pactadas }\end{array}$ \\
\hline $\begin{array}{l}\text { Comparación por notas } \\
\text { obtenidas }\end{array}$ & $\begin{array}{l}\text { Se construye una interfaz donde el estudiante puede ver la evaluación de } \\
\text { sutrabajo en comparación con el promedio del curso }\end{array}$ \\
\hline $\begin{array}{l}\text { Registro de actividad } \\
\text { participación }\end{array}$ & $\begin{array}{l}\text { Interfaz para visualizar la actividad del estudiante y realizar una } \\
\text { comparación frente a la media del grupo. }\end{array}$ \\
\hline Plan de estudios & Se refleja visualmente las asignaturas cursadas, aprobadas y reprobadas. \\
\hline
\end{tabular}

\section{Conclusiones y futuros trabajos}

Utilizar una metodología inclusiva para el desarrollo del proyecto ha permitido tener un visión global del problema a la vez que se consideran diferentes perspectivas de solución dadas por todos los involucrados, este esquema de trabajo promueve la generación de ideas de que alguna forma los diseñadores y desarrolladores han incorporado en las nuevas interfaces.

Involucrar en las interfaces elementos afectivos que reflejen el estado del sistema frente a la percepción emocional de los actores del proceso ha generado diversas reacciones que el equipo de trabajo considera positivas, entre ellas tenemos: el docente o estudiante tiene la percepción de acompañamiento en su proceso, algunos actores se ven motivados a trabajar cuando ven que el sistema muestra un descenso en su producción, las comparaciones realizadas por el sistema frente a la producción de los diferentes actores se torna incomoda sin embargo genera una reacción competitiva que 
en la mayoría de los casos es positiva, las interfaces reflejan un espacio de trabajo lúdico pero a la vez provocador.

Se prevé la construcción de estudios de caso que permita validar cada uno de los elementos incorporados en las interfaces de los aplicativos, a la vez que se considera involucrar elementos de análisis automáticos orientados a caracterizar el comportamiento de un usuario a lo largo del proceso y por ende elementos que contribuyan con la estimulación de su proceso.

\section{Referencias}

Almeida L., Baranauskas M. C. (2008). Awareness em Sistemas Colaborativos: Conceitos y Desafios, Relatorio Técnico nro. 23. Insitituto de Computación, Universidad de Campinas.

E-Cidadania, Sistemas e Métodos na Constituição de uma Cultura mediada por Tecnologias de Informação e Comunicação, disponível em http://styx.nied.unicamp.br:8080/ecidadania, último acesso junho 2013.

Hornung, H. H.; Almeida, L. D. A.; Melo-Solarte, D. S. Lições aprendidas no desenvolvimento de software social. In: Baranauskas, M.C.C.; Martins, M.C.; Valente, J.A.. (Org.). Codesign de Redes Digitais - Tecnologia e Educação a Serviço da Inclusão Social. 1ed.Porto Alegre: Penso Editora Ltda., 2013, p. 174-195.

Koch, A. S. (2005). Agile Software Development: Evaluating the Methods for Your Organization. Artech House, Norwood, MA, USA.

Liu, K. (2000) Semiotics in information systems engineering. Cambridge University Press, New York, NY, USA.

Longaray A. N. C., Behar P. A., Longhi M. T. (2012) Afetividade em um ambiente virtual de aprendizagem: um estudo sobre os indicadores pedagógico. In: Anais do 23 Simpósio Brasileiro de Informática na Educação (SBIE 2012). Rio de Janeiro, Brasil

Longhi, M. T., Behar, P. A., Bercht, M.; Simonato, G. (2010) "Os fatores motivacionais e os estados de ânimo em ambientes virtuais de aprendizagem". In: TISE 2010. Santiago do Chile, Chile, Dez. 1-3, p. 551-558.

Melo-Solarte D. S. \& Baranauskas M. C. (2009). Aprendizagem Colaborativa Baseada em Problemas (ACBP): Modelo Conceitual e Ferramentas. Dissertação de mestrado. Instituto de Computação. Universidade Estadual de Campinas. Brasil.

Neris, V. P. (2010) Estudo e proposta de um framework para o design de interfaces de usuario ajustáveis. PhD thesis. Instituto de computação, Universidade de Campinas. Brasil.

Stamper, R. (1993). "Social Norms in requirements analysis an outline of MEASUR". In Requirements Engineering, Technical and Social Aspects. Academic Press.

Zorrilla, M. e Álvarez, E (2008). MATEP: Monitoring and Analysis Tool for ELearning Platforms, Proceedings of the 2008 Eighth IEEE International Conference on Advanced Learning Technologies, p.611-613. 